\title{
DESEMPENHO DA CO-KRIGAGEM NA DETERMINAÇÃO DA VARIABILIDADE DE ATRIBUTOS DO SOLO ${ }^{(1)}$
}

\author{
João Carlos Angelico ${ }^{(2)}$
}

\begin{abstract}
RESUMO
A agricultura de precisão requer princípios de manejo de acordo com a variabilidade no campo, exigindo técnicas eficientes para estimar e mapear a variabilidade espacial e, ou, temporal das características e propriedades dos solos. Uma análise detalhada dos atritubos físicos e químicos do solo demanda custos e tempo relativamente elevados. Por esse motivo, costuma-se aplicar métodos estatísticos de interpolação para obter as características e propriedades dos solos nos locais não amostrados, visando diminuir o número de amostras necessárias para um bom mapeamento do campo. Algumas variáveis que caracterizam os atributos de determinado solo são, freqüentemente, onerosas e de difícil execução. Nestas situações, é interessante estimar tais variáveis com base em outras que apresentam boa correlação espacial com as primeiras e de simples determinação. Isto pode ser feito por meio de um semivariograma cruzado. $O$ interpolador que utiliza o semivariograma cruzado em sua modelagem é chamado de co-krigagem . O objetivo deste trabalho foi investigar a eficiência do método estatístico da cokrigagem na estimativa do pH e do Mn de acordo com a matéria orgânica, obtendo as margens de erros associadas a esta técnica por comparação dos valores estimados com aqueles determinados em laboratório. Os resultados mostraram que a cokrigagem foi capaz de estimar as variabilidades dos solos com grande exatidão.
\end{abstract}

Termos de indexação: geoestatística, variabilidade espacial, agricultura de precisão.

\footnotetext{
${ }^{(1)}$ Recebido para publicação em julho de 2005 e aprovado em setembro de 2006 .

(2) Professor de Estatística da Faculdade Origenes Lessa - FACOL. Rod. Osny Matheus, Km 08, CEP 18683-900 Lençóis Paulista (SP). E-mail: jcangelico@facol.br
} 


\title{
SUMMARY: CO-KRIGING PERFORMANCE IN THE DETERMINATION OF VARIABILITY OF SOIL ATTRIBUTES
}

\begin{abstract}
In precision agriculture management principles must be adapted to the field variability. This requires efficient techniques to estimate and map the spatial and/or temporary variability of soil attributes and properties. However, the determination of some variables that characterize the properties of a particular soil is often onerous and troublesome. In these situations it is interesting to estimate such variables as a function of others that present good space correlation with the forner and are of simpler determination. This is possible by a cross-semivariogram. The interpolator that uses the cross-semivariogram in modeling is called co-kriging. The aim of this study was to test the co-kriging method for estimating $\mathrm{pH}$ and $\mathrm{Mn}$ as related to soil organic matter to obtain the error range associated to this technique and to compare the estimated values with those determined in laboratory. Results showed that soil variability can be estimated with high precision by co-kriging.
\end{abstract}

Index terms: geostatistics, spatial variability, precision agriculture.

\section{INTRODUÇÃO}

Na literatura, encontram-se trabalhos inerentes à variabilidade espacial e temporal das propriedades dos solos, publicados no início do século XX, tais como: os de Montgomery (1913), Robinson \& Lloyd (1915) e Pendleton (1919), citados por Vieira et al (1983). De início, as análises estatísticas de dados consideravam as variáveis aleatórias independentes entre si, ou seja, supunham que as observações vizinhas não exerciam influências umas sobre as outras. Contudo, fenômenos naturais apresentam-se freqüentemente com certa estruturação entre vizinhos. Desta forma, pode-se dizer que as variações não ocorrem ao acaso, apresentando certo grau de dependência espacial. Foi o engenheiro de Minas Sul-Africano, Daniel G. Krige, no ano de 1951, que, com seus trabalhos com dados de mineração, concluiu que a variação daqueles dados apresentava uma estruturação que dependia da distância de amostragem e, desta constatação, surgiram os conceitos básicos de geoestatística. Na geoestatística, leva-se em consideração a variabilidade espacial e, ou, temporal de dados, complementando, ou mesmo substituindo, eficientemente, a análise estatística clássica. Um requisito básico para aplicação dos métodos geoestatísticos é a necessidade de referenciamento das amostras, seja com relação às coordenadas geográficas, seja com relação ao tempo (Zimback, 2003).

Numerosos estudos feitos por Kravchenko \& Bullock, (1999) demonstram que a ponderação do inverso da distância é mais fácil de se realizar, enquanto a Krigagem consome mais tempo e é mais complicada de se aplicar. Por outro lado, a Krigagem faz uma descrição mais acurada da estrutura espacial dos dados e produz valiosa informação sobre a distribuição da estimativa do erro.

Creutin \& Obled (1982) e Tabios \& Salas (1985) fizeram comparações entre algumas técnicas de interpolação e a Krigagem para a distribuição da precipitação anual. A Krigagem mostrou-se mais eficiente. Warrick et al. (1988) obtiveram melhores resultados para a Krigagem, quando a compararam com o método do inverso da distância, para mapear a produtividade de tomate e algumas propriedades do solo. Laslett et al. (1987) conseguiram resultados mais satisfatórios na obtenção do $\mathrm{pH}$ do solo, usando a Krigagem, quando comparada com o método do inverso da distância.

Entretanto, existem trabalhos que demonstram ser o método do inverso da distância mais eficiente que a Krigagem. Weber \& Englund (1992) e Wollenhaupt et al. (1994), para mapear a disponibilidade de $\mathrm{P}$ e K no solo, demonstraram superioridade no método do inverso da distância. Também Gotway et al. (1996) mostraram maior eficiência do método do inverso da distância no mapeamento do teor de matéria orgânica $\mathrm{e} \mathrm{NO}_{3}{ }^{-}$no solo.

Fietz et al. (2000) avaliaram a variabilidade espacial e confeccionaram mapas de necessidade de calcário de uma área de Latossolo Vermelho, localizada na Embrapa Agropecuária Oeste (em Dourados - MS), com base em técnicas de geoestatística. Por meio de um semivariograma dos dados, confeccionou-se uma malha utilizando a técnica de Krigagem. Com os valores interpolados, elaborou-se um mapa de aplicação localizada de calcário. Os dados de necessidade de calcário apresentaram dependência espacial com nível moderado, com efeito pepita e patamar de $27 \%$ e alcance de $165 \mathrm{~m}$. Com esses resultados, a geoestatítica mostrou-se valiosa na interpolação de dados e, conseqüentemente, na confecção de mapas utilizados na agricultura de precisão.

Torres \& Prochnow (2000) utilizaram uma combinação dos softwares "Fieldlink", para a coleta dos dados no campo, e "Aglink", para a elaboração dos mapas. Inicialmente, foram elaborados os mapas com amostras de solos coletadas sob a "saia" da cultura de 
citrus e, em seguida, elaborou-se outro mapa com amostras de solo coletadas no meio da rua. Os mapas gerados mostraram $\mathrm{pH}$ mais elevado e teores de nutrientes menores no meio da rua, decorrentes, possivelmente, da aplicação localizada de fertilizantes sob a copa das plantas. O trabalho mostrou ser o mapeamento da fertilidade do solo importante ferramenta para auxiliar agrônomos, técnicos e agricultores a elaborar uma melhor estratégia de calagem e de adubação, porém os autores levantam a delicada questão sobre o número ideal de subamostras que deve compor uma amostra, o raio de retirada das subamostras e o local, dentro da grade de amostragem, onde se deve fazer a amostragem.

Vieira et al. (2000) analisaram a variabilidade espacial de algumas características do solo e a produção de culturas anuais, em três locais do Estado de São Paulo, pela geoestatística. As amostras foram coletadas em grades de 10 por $10 \mathrm{~m}$. Observou-se que as variabilidades do teor de $\mathrm{C}$ e de $\mathrm{Na}$ eram extremamente contínuas, com semivariogramas esféricos. O semivariograma não representou adequadamente a elevada variabilidade das determinações ligadas à textura do solo, tampouco se observou relação entre a variabilidade das características ligadas à permeabilidade do solo e a variabilidade da espessura do solo.

Marques Júnior (2000) avaliou a variabilidade espacial das características granulométricas e químicas dos solos e da produção de café em diferentes superfícies geomórficas, sobre solos altamente intemperizados. Os resultados obtidos foram:

- as características químicas e granulométricas revelavam dependência com o relevo, mesmo de pequena expressão e em solos altamente intemperizados e manejados quimicamente;

- todos os atributos químicos e granulométricos apresentaram dependência espacial;

- os resultados de semivariogramas foram importantes para subsidiar informações sobre a gênese e evolução dos solos na paisagem;

- a produção de café apresentou dependência espacial em ambas as superfícies, bem como alcance maior na superfície geomórficas mais plana.

Em algumas situações é interessante estimar determinada variável do solo em relação a outra, cujo custo e, ou, tempo de determinação é menor. Isto pode ser feito por meio de um semivariograma cruzado (a co-krigagem) (Guimarães, 2004).

Carvalho \& Queiroz (2002) utilizaram a cokrigagem para estudar a variabilidade espacial de alguns atributos físicos de um Latossolo Vermelho distrófico, estabelecido sob preparo convencional e cultivado com o feijoeiro comum durante o ano agrícola de 1999/2000, com vistas em fornecer subsídios para o planejamento da sua área agrícola experimental.
Os atributos estudados foram: macroporosidade (MA), microporosidade (MI), porosidade total (PT), densidade do solo (DS), resistência à penetração (RP) e umidade do solo (UG). A coleta dos dados foi efetuada com os pontos amostrais dispostos segundo uma malha com 103 pontos, nas profundidades de 0-5 $\mathrm{cm}$ (superfície) e 15-20 cm (subsuperfície). Os resultados revelaram que a variabilidade pesquisada foi: (a) muito alta, para a RP, em ambas as profundidades, e, para a MA, na subsuperfície; (b) alta, para a MA, na superfície; (c) média, para a UG, em ambas as profundidades, e (d) baixa, para a MI, PT e DS, em ambas as profundidades. Todos os atributos do solo apresentaram dependência espacial, com exceção da MI na superfície. Desta forma, na camada superficial, a PT, DS, RP, MA e UG obtiveram, respectivamente, os alcances de 8,6; 13,$1 ; 15,2 ; 15,8$ e 18,4 m. Já, na subsuperfície, a DS, $\mathrm{UG}, \mathrm{RP}, \mathrm{MI}, \mathrm{MA}$ e PT atingiram, respectivamente, os alcances de 1,$0 ; 9,1 ; 14,4 ; 18,4 ; 19,2$ e $23,7 \mathrm{~m}$.

Chig et al. (2004) utilizaram os valores de $\mathrm{pH}$ para estimar pela co-krigagem a distribuição espacial do carbono orgânico dos solos em quatro microbacias sob vegetação de floresta no estado de Mato Grosso.

Couto \& Klamt (1999) estimaram a deficiência de Mn pela co-krigagem em um solo sob pivô central na profundidade de 0-20 cm, em uma plantação de milho no Sul do Estado de Mato Grosso.

Neste trabalho, pretendeu-se verificar o desempenho da co-krigagem na estimativa do $\mathrm{pH}$ e do teor de Mn de acordo com o teor de matéria orgânica.

\section{MATERIAIS E MÉTODOS}

Os dados de solos (consta de uma planilha com 275 dados) foram obtidos junto à Serrana Fertilizantes do grupo Bunge, que efetuou, em seus próprios laboratórios, a determinação da $\mathrm{MO}$, do $\mathrm{pH}$ e do $\mathrm{Mn}$.

As amostras de solos foram coletadas em uma região próxima à divisa dos estados de Mato Grosso e Mato Grosso do Sul, no município de Itiquira, nas coordenadas compreendidas entre $17^{\circ} 35^{\prime} 22$ " e $17^{\circ} 42^{\prime} 38$ "de latitude Sul e $54^{\circ} 61^{\prime} 75^{\prime}$ " e $54^{\circ} 69$ ' $56 "$ de longitude Oeste. A região apresenta clima tropical quente e úmido, com precipitação média anual de $1.500 \mathrm{~mm}$, com intensidade máxima em dezembro, janeiro e fevereiro. A temperatura média anual é de $22^{\circ} \mathrm{C}$. As amostras foram coletadas a uma profundidade de $0-20 \mathrm{~cm}$ em uma área com pastagem há mais de 5 anos.

As amostras de solo foram coletadas, secas ao ar e peneiradas em malha de $2 \mathrm{~mm}$ (TFSA). Na determinação do $\mathrm{pH}$, foram utilizados $10 \mathrm{~cm}^{3}$ de TFSA misturados com $25 \mathrm{~cm}^{3}$ de $\mathrm{CaCl} 0,01 \mathrm{~mol} \mathrm{~L}^{-1}$, conforme Tomé Júnior (1997). O carbono orgânico foi determinado pelo método Walkley-Black, enquanto o Mn foi extraído com o extrator Mehlich-1, e 
posteriormente dosado por espectroscopia de absorção atômica (Camargo et al., 1986).

Dos 275 dados existentes na planilha, selecionaramse, aleatoriamente, 28 (aproximadamente $10 \%$ das amostras), que foram utilizados, como referência, na verificação da eficiência da co-krigagem. As 247 amostras restantes foram utilizadas nas estimativas.

A co-krigagem foi desenvolvida com auxílio do softwre $\mathrm{GS}^{+}$(Gamma Design Software), Versão Beta (5.0.3).

\section{RESULTADOS E DISCUSSÃO}

Não foi observada concentração de valores em posições específicas da área, tampouco ocorreu sentido preferencial na distribuição dos dados. Tal fato é um bom indicativo de que a distribuição espacial das variáveis, nesta área, é aleatória e isotrópica (Cambardella et al., 1994).

A análise descritiva geral para as variáveis analisadas é apresentada no quadro $1 . \mathrm{O}$ pH, seguido da $\mathrm{MO}$, revelou maior uniformidade (menor coeficiente de variação) do que o Mn. Os coeficientes de assimetria e curtose mostraram que a $\mathrm{MO}$ e o $\mathrm{pH}$ apresentaram distribuições simétricas e ligeiramente platicúrticas, enquanto o $\mathrm{Mn}$ revelou discreta assimetria à direita $\mathrm{e}$ distribuição normal (Guimarães, 2004).

A figura 1 mostra o semivariograma da matéria orgânica. Notou-se que a matéria orgânica apresentou dependência espacial que pode ser descrita pelo modelo esférico com alcance de $103 \mathrm{~m}$, ou seja, amostras da matéria orgânica selecionadas a distâncias inferiores a $103 \mathrm{~m}$ foram correlacionadas entre si. O efeito pepita apresentou valor de 0,0729 e o patamar de 0,1468. A relação entre o efeito pepita e o patamar de 49,66 \% indicou ser a dependência espacial moderada (Cambardella, 1994).

Nos semivariogramas, tanto para o $\mathrm{pH}$ como para o Mn, obteve-se o melhor ajuste com o modelo exponencial (Figura 1). Os alcances foram de $51 \mathrm{~m} \mathrm{e}$ $48 \mathrm{~m}$, para o $\mathrm{pH}$ e o $\mathrm{Mn}$, respectivamente. De acordo com Cambardella (1994), a dependência espacial para as duas variáveis mostrou-se forte, uma vez que a relação entre o efeito pepita e o patamar foram iguais a $11,21 \%$, para o $\mathrm{pH}$, e a $15,33 \%$, para o $\mathrm{Mn}$.

Analisando a relação linear entre o pH e a matéria orgânica, obteve-se um coeficiente de correlação linear de 0,926 , indicando a forte associação positiva entre o pH e a matéria orgânica (Spiegel, 1985) e, portanto, estimativas do $\mathrm{pH}$ podem ser feitas com base no teor de MO. Entre os teores de Mn e a MO, obteve-se um coeficiente de correlação linear de 0,764, indicando menor associação positiva do que no caso do $\mathrm{pH}$, porém, ainda assim, foi possível utilizar o teor de MO como co-variável na estimativa do Mn.

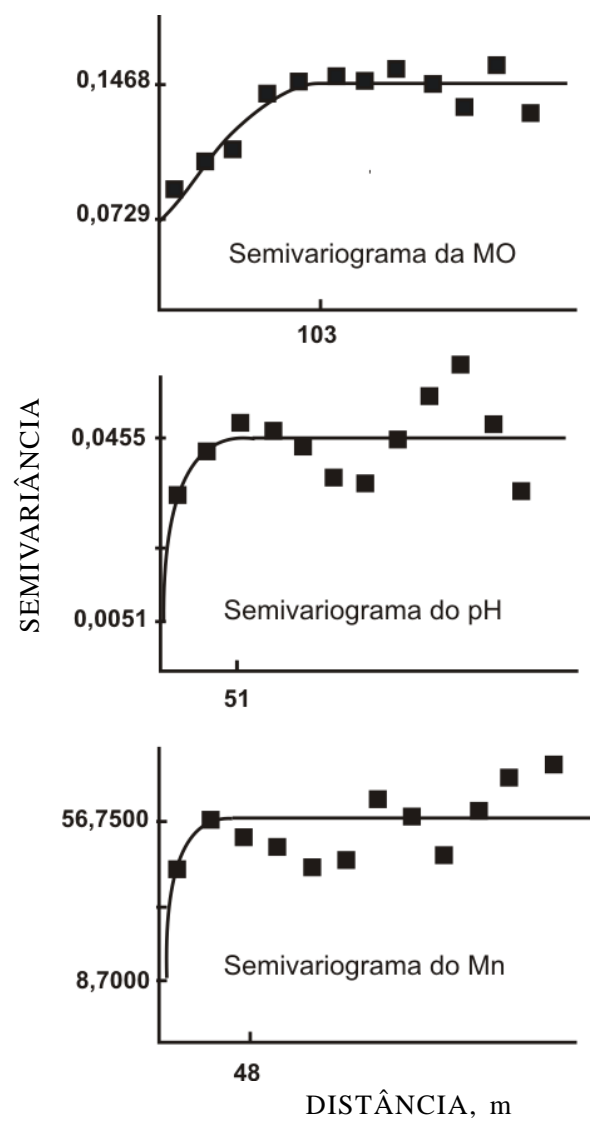

Figura 1. Semivariogramas de teor de matéria orgânica, de pH e do teor de manganês.

Quadro 1. Análise descritiva das variáveis analisadas

\begin{tabular}{|c|c|c|c|}
\hline \multirow{2}{*}{ Estatística } & \multicolumn{3}{|c|}{ Atributo } \\
\hline & MO & $\mathbf{p H}$ & Mn \\
\hline & dag $\mathrm{kg}^{-1}$ & & $\mathrm{mg} \mathrm{dm} \mathrm{m}^{-3}$ \\
\hline Média & 3,62 & 5,10 & 24,54 \\
\hline Mediana & 3,70 & 5,10 & 23,20 \\
\hline Moda & 3,80 & 5,20 & 22,00 \\
\hline Desvio-padrão & 0,36 & 0,21 & 7,20 \\
\hline Coeficiente de variação (\%) & 9,96 & 4,06 & 29,36 \\
\hline Coeficiente de assimetria & 0,60 & $-0,17$ & 3,27 \\
\hline Coeficiente de curtose & $-0,19$ & 1,07 & 4,14 \\
\hline
\end{tabular}


A figura 2 ilustra os semivariogramas cruzados do $\mathrm{pH}$ e do $\mathrm{Mn}$, respectivamente, usando, como covariável, o teor de MO.

Para avaliar semivariograma cruzado do $\mathrm{pH}$, as estimativas dos parâmetros do modelo esférico foram: alcance de $13 \mathrm{~m}$, efeito pepita de 0,00258 e patamar de 0,00898. Verificou-se que a utilização do teor de $\mathrm{MO}$, como uma co-variável para a estimativa do $\mathrm{pH}$, provocou alteração no alcance da dependência espacial, mas, ainda assim, verificou-se que a correlação espacial deve ser considerada para a realização das estimativas. Segundo Guimarães (2004), essa alteração pode estar relacionada com os modelos individuais diferenciados. O semivariograma cruzado do teor de Mn também se ajustou melhor ao modelo esférico. Observou-se que o alcance foi de $21 \mathrm{~m}$ com efeito pepita de 0,1950 e patamar de 0,2349.

O quadro 2 permite uma comparação direta entre os valores estimados pela co-krigagem e os valores medidos em laboratório (valor real), seguidos dos respectivos erros. Os erros foram determinados da seguinte forma: Erro $=\left|\left(\frac{100 \text { Valor Estimado }}{\text { Valor Real }}\right)-100\right|$

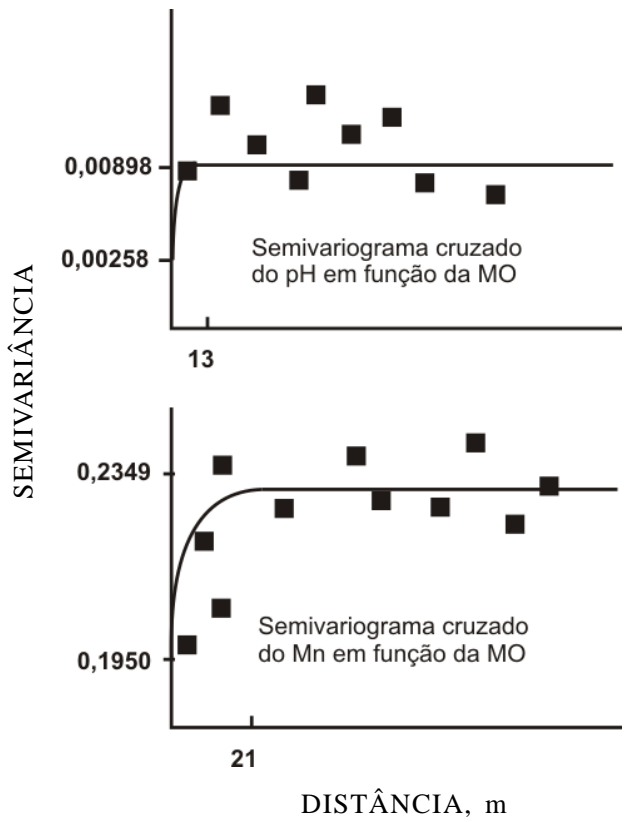

Figura 2. Semivariogramas cruzados do pH e do teor de manganês, considerando a MO como covariável.

Quadro 2. Comparação entre os valores estimados e os valores reais de pH e teores de manganês

\begin{tabular}{|c|c|c|c|c|c|}
\hline \multicolumn{2}{|c|}{$\mathrm{pH}\left(\mathrm{CaCl}_{2}\right)$} & \multirow{2}{*}{ Erro } & \multicolumn{2}{|c|}{ Mn } & \multirow{2}{*}{ Erro } \\
\hline Real & Estimado & & Real & Estimado & \\
\hline & & $\%$ & \multicolumn{2}{|c|}{$\mathrm{mg} \mathrm{dm}^{-3}$} & $\%$ \\
\hline 5,20 & 5,09 & 2,25 & 17,10 & 17,16 & 0,33 \\
\hline 4,80 & 5,03 & 4,75 & 19,90 & 20,57 & 3,37 \\
\hline 5,10 & 5,15 & 0,92 & 22,70 & 28,06 & 23,60 \\
\hline 5,30 & 5,27 & 0,60 & 24,00 & 32,76 & 36,51 \\
\hline 5,00 & 5,15 & 3,08 & 31,20 & 30,71 & 1,57 \\
\hline 5,20 & 5,20 & 0,00 & 20,50 & 23,36 & 13,93 \\
\hline 5,20 & 5,16 & 0,69 & 26,50 & 23,84 & 10,03 \\
\hline 5,20 & 5,50 & 0,98 & 17,80 & 21,12 & 18,65 \\
\hline 5,20 & 5,04 & 3,06 & 17,00 & 17,49 & 2,90 \\
\hline 5,10 & 5,16 & 1,18 & 17,70 & 18,74 & 5,87 \\
\hline 4,80 & 4,87 & 1,50 & 15,60 & 17,26 & 10,67 \\
\hline 5,20 & 5,01 & 3,58 & 19,30 & 18,76 & 2,81 \\
\hline 5,00 & 5,15 & 3,04 & 28,90 & 33,43 & 15,66 \\
\hline 5,40 & 5,17 & 4,20 & 20,40 & 24,55 & 20,32 \\
\hline 5,00 & 5,10 & 2,06 & 20,60 & 28,28 & 37,27 \\
\hline 5,30 & 5,25 & 0,94 & 24,40 & 24,03 & 1,53 \\
\hline 5,20 & 5,22 & 0,46 & 26,40 & 26,33 & 0,26 \\
\hline 5,20 & 5,18 & 0,46 & 20,50 & 21,48 & 4,80 \\
\hline 5,70 & 5,19 & 9,03 & 25,70 & 22,72 & 11,58 \\
\hline 5,30 & 5,25 & 0,98 & 21,10 & 21,59 & 2,31 \\
\hline 5,20 & 5,21 & 0,27 & 22,40 & 23,24 & 3,75 \\
\hline 5,30 & 5,21 & 1,62 & 22,60 & 23,48 & 3,89 \\
\hline 5,00 & 4,78 & 4,34 & 19,10 & 20,49 & 7,26 \\
\hline 4,90 & 4,90 & 0,08 & 33,10 & 30,44 & 8,03 \\
\hline 5,00 & 5,02 & 0,48 & 29,60 & 26,30 & 11,14 \\
\hline 4,80 & 4,96 & 3,34 & 25,10 & 29,16 & 16,19 \\
\hline 5,00 & 4,93 & 1,34 & 24,00 & 25,41 & 5,89 \\
\hline 5,30 & 5,10 & 3,77 & 28,30 & 20,30 & 28,27 \\
\hline Erro Médio & & 2,11 & & & 11,01 \\
\hline
\end{tabular}


Dentre as 28 amostras selecionadas para verificação, observou-se que o erro maior na estimativa do $\mathrm{pH}$ foi de 9,04 e, para o teor de Mn de 37,27. Os resultados foram bastante satisfatórios quando se analisou a média dos erros que, para o $\mathrm{pH}$, ficou em torno de $2 \%$ e, para o teor de Mn, em torno de $11 \%$, resultados significativos para a maioria das práticas agrícolas (McLellan e Friesen, 1997).

\section{CONCLUSÕES}

1. A utilização da co-krigagem possibilitou maior eficiência da caracterização química do solo por meio da redução de amostras com a utilização de uma covariável.

2. As margens de erros apresentadas nas estimativas mostraram-se aceitáveis para a maioria das práticas agrícolas, incluindo a agricultura de precisão, como, por exemplo, a aplicação de corretivos a taxas variáveis no solo.

3. Os resultados mostraram que a co-krigagem pôde realizar a estimativa do $\mathrm{pH}$ e do $\mathrm{Mn}$ eficientemente, usando, como co-variável, o teor de MO.

\section{LITERATURA CITADA}

CAMARGO, O.A.; MONIZ, A.C.; JORGE, J.A. \& VALADARES, J.M.A.S. Métodos de análise química, mineralógica e física de solos do Instituto Agronômico de Campinas. Campinas, Instituto Agronômico de Campinas. Campinas, instituto Agronômico, 1986. 94p. (Boletim Técnico, 106)

CAMBARDELLA, C.E.; MOORMAN, T.B.; NOVAK, J.M.; PARKIN, T.B.; KARLEN, D.L.; TURCO, R.F. \& KONOPKA, A.E. Field-scale variability of soil properties in Central Iowa soils. Soil Sci. Soc. Am. J., 58:1501-1511, 1994.

CARVALHO, J.R.P. \& QUEIROZ, E.F. Uso da co-krigagem colocalizada na determinação da distribuição espacial de precipitação. Embrapa Informática Agropecuária. Área de Comunicação e Negócios. Campinas. $1^{\mathrm{a}}$ edição on line, 2002 .

CHIG, L.A.; COUTO, E.G.; RIHA, S.; JOHNSON, M.; NOVAES, J.P.; LEHMANN, J. \& SELVA, E.C. Uso da Krigagem ordinária e da co-krigagem para estimar a matéria orgânica em solos de quatro microbacias sob vegetação de floresta no município de Juruena, MT. In: COFERÊNCIA CIENTÍFICA DO LBA, 2004, Brasília, Anais. Brasília, 2004. p.48-54.

COUTO, E.G. \& KLAMT, E. Variabilidade espacial de micronutrientes em solo sob pivô central no sul do Estado de Mato Grosso. Pesq. Agropec. Bras., 34:2331-2339, 1999.

CREUTIN, J.D. \& OBLED, C. Objetive analysis and mapping techniques for rainfalls fields: An objetive comparison. Water Res. Res., 18:413-431, 1982.
FIETZ, C.R.; FABRICIO, A.C. \& SALTON, J.C. Mapa de aplicação localizada de calcário em uma área experimental. In: BALASTREIRE, L.A. O estado da arte da agricultura de precisão no Brasil. Piracicaba, ESALQ, 2000. p.165169.

GOTWAY, C.A. \& HARTFORD, A.H. Geostatistical methods for incorporating auxiliary information in the prediction of spatial variables. J. Agric., Biol. Environ. Statis., 1:1739, 1996.

GUIMARÃES, E.C. Geoestatística básica e aplicada. Uberlândia, Universidade Federal de Uberlândia, 2004. 77p.

KRAVCHENKO, A.N. \& BULLOCK, D.G. A comparative study of interpretation methods for mapping properties. Agron. J., 91:393-400, 1999.

LASLETT, G.M.; McBRATNEY, A.B.; PAHL， P. \& HUTCHINSON, M. Comparison of several spatial prediction methods for soil pH. J. Soil Sci., 38:325-341, 1987.

MARQUES Jr., J. Variabilidade espacial de propriedades químicas e físicas de Latosssolos em áreas de Cerrado sob cultivo de café, em Patrocínio, MG. In: BALASTREIRE, L.A. O estado da arte da agricultura de precisão no Brasil. Piracicaba, ESALQ, 2000. p.165-169.

McLELLAN, J.F. \& FRIESEN, L. Pulsearch Navigation Systems Inc. Calgary, Alberta, Canada, 1997.

SPIEGEL, M.R. Estatistica. 2.ed. São Paulo, Schaum McGrawHill, 1985. p.298-308.

TABIOS, G.Q. \& SALAS, J.D. A comparative analysis of techniques for spatial interpolation of precipitation. Water Res.. Bull., 21:365-380, 1985.

TORRES, F.P. \& PROCHNOW, L.I. Mapeamento de fertilidade do solo. BALASTREIRE, L.A. O estado da arte da agricultura de precisão no Brasil. Piracicaba, ESALQ, 2000. p.165-169.

VIEIRA, S.R.; HATFIELD, D.R.; NIELSEN, D.R. \& BIGGAR, J.W. Geostatistical theory and application to variability of some agronimical properties. Hilgardia, 31:75, 1983.

VIEIRA, S.R.; DECHEN, S.C.F.; MARIA, I.C.; MARTINS, A.L.M. \& BOTOLETTO, N. Mapeamento de Atributos de Solo e Planta Usando Geoestatística. Artigo apresentado no trabalho de Balastreire, L.A. O estado da arte da agricultura de precisão no Brasil. Piracicaba, ESALQ, 2000. p.165-169.

WARRICK; A.W.; ZHANG, R.; HARRIS, M.K. \& MYERS, D.E. Direct comparations between kriging and other interpolation - Validation of flow and transport models for the unsaturated zone. New Mexico, 1988. p.254-326.

WEBER, D.D. \& ENGLUND, E.J. Evalution and comparison of spatial interpolators. Math.Geol., 24:381-391, 1992.

WOLLENHAUPT, N.C.; WOLKOWSKI, R.P. \& CLAYTON, M.K. Mapping soil test phosphorus and potassium for variable-rate fertilizer application. J. Prod. Agric., 7:441448, 1994.

ZIMBACK, C.R.L. Geoestatística. Grupo de estudos e pesquisas agrárias georreferenciadas. Botucatu, Universidade Estadual Paulista, 2003. p.1-25 